\title{
Passive optoelectronics systems for standoff gas detection: results of tests
}

\author{
M. Kastek ${ }^{1}$, T. Piątkowski ${ }^{1}$, P. Lagueux ${ }^{2}$, M. Chamberland ${ }^{2}$ \\ \& M. A. Gagnon ${ }^{2}$ \\ ${ }^{1}$ Institute of Optoelectronics, Military University of Technology, Poland \\ ${ }^{2}$ Telops Inc., Québec, Canada
}

\begin{abstract}
The paper presents a review of optoelectronic systems for stand-off detection of gases. Selected passive devices are presented, including thermal cameras with modified optics for remote gas detection and imaging Fourier transform spectrometers. The latter ones are capable of remote measurements of spectral characteristics of chemical compounds and, after further data analysis, they provide gas identification capabilities and even concentration estimates. This paper presents detection and identification of gases using an infrared imaging Fourier-transform spectrometer. The principle of operation of the spectrometer and the method for gases detection and identification is shown in the paper. The new software with implementation of method based on Clutter Match Filter, focused on detection and identification of gases is presented. Some results of the detection of various types of gases are also given.

Keywords: gas detection, hyperspectral detection, stand-off detection.
\end{abstract}

\section{Introduction}

A problem of remote detection of chemical substances appears in many, sometimes extremely different fields of human activities. Applications of detection devices include monitoring of technological processes, diagnostics of industrial installations, monitoring of natural environment, and military purposes. Such diversity has caused development of many detection methods employing various physical phenomena due to which detection and identification of chemical compounds are possible. Among these methods, a significant group constitutes the solutions using a phenomenon of selective absorption of electromagnetic radiation 
by chemical compounds. Here, we can distinguish the solutions based on absorption of laser radiation emitted by an illuminator, being an element of a measuring system, and thermovision methods recording thermal radiation. The methods of the first group are the active methods employing absorption of laser radiation by chemical compounds. Thermovision methods are based on using the absorption bands of chemical compounds in infrared.

Thermovision methods, employing arrays detectors, provide an image (in real time) of the observed scenery with the marked regions of searched chemical substances. Usefulness of this method results from the fact that a lot of substances and chemical compounds have their absorption bands in infrared.

The devices operating in infrared, used for detection of chemical substances (gases) in the atmosphere have spectral characteristics and resolution matched to the absorption bands of compounds to be detected. Two types of such devices can be distinguished. One, it is a system similar to typical thermal camera but additionally equipped with the filter system ensuring the required spectral resolution and the signal analysis system. The other type includes the device based on the principles of Fourier spectroscopy (Fourier Transform Infrared Spectroscopy - FTIR) that is expensive and so not readily available.

\section{Passive systems and devices for stand-off gas detection}

Thermal cameras used for detection of volatile chemical substances have to be equipped with the systems ensuring adequate spectral resolution. It is connected with the necessity of application of the filters (usually tunable ones, increasing universality of a device), the systems of signal analysis, and often special optical systems.

Example solution of application of a thermal camera for gas detection, equipped with an optical filter, is schematically presented in Fig. 1. The system consists of: an Agema THV900LW camera equipped with the bandpass filters having characteristics matched to the absorption bands of gases, a Cassegrainian telescope, frame grabber, CCD camera, and PC computer with special software for analysis. The IR camera is equipped with suitable interference filters, used to isolate a small spectral region containing absorption features of the gas. The image processing was performed according to the following scheme: A and B are captured at the same time using the Cassegrainian telescope by the IR camera and frame grabber ( $A$ - image of the infrared scene from one of telescope openings, $B$ - image of the same scene with gas cell in front of the opening telescope), next an error normalization of image from the Cassegrainian is created to handle imperfections such as symmetrical vignetting and stray-light from the two openings ( $E=A_{0} / B_{0}$ where $A_{0}, B_{0}$ are images registered with no gas in the scene) after this images are digitally overlapped by translation, optimization within the region of gas and gas correlation images is calculated as $G=A / B / E$. In this way the appropriate offset is subtracted from the images. This image, which presents the region of gas presence in the observed scenery, is superposed on an image from CCD camera [1]. 


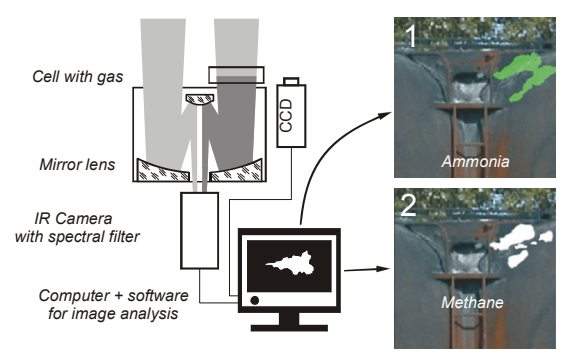

Figure 1: Gas detection system with a thermal camera [1].

A result of these operations is shown in a picture situated next to the camera system (Fig. 2). Image (a) presents the total leakage of ammonia. Due to filling up the gas chamber with methane, detection of this substance and its visualization is possible - image (b).

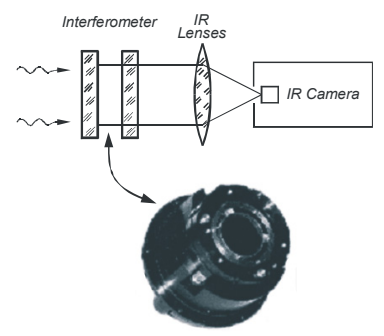

(a)

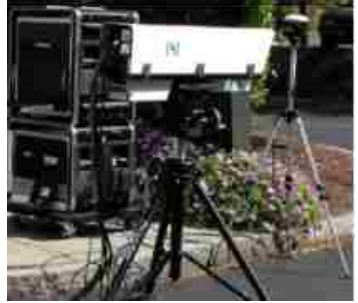

(b)

Figure 2: The scheme of thermal camera and Fabry-Perot interferometer [2].

One method of a thermal camera application for searching gas leakages is a thermal camera system equipped additionally with a Fabry-Perot interferometer. An interferometer in IR system plays a role of a tunable optical filter (Fig. 2).

It selects the wavelength of IR radiation illuminating, at the given moment, the pixels of FPA of a thermal camera. An operation range of the interferometer is $3-$ $5 \mu \mathrm{m}$ (MWIR version) or 8-12 $\mu \mathrm{m}$ (LWIR) and tuning velocity is of about 10-20 ms. An interferometer module, containing a detector matrix, has been developed by the Physical Sciences, Inc. and it is known as AIRIS (Adaptive InfraRed Imaging Spectroradiometer) [2].

By applying the aforementioned tunable optical filters it is possible to create a gas detection system around a thermal camera. The filter is tuned to the absorption band of a chosen compound and the interpretation of thermal image is performed by a human operator. The gas cloud appears as a "black smoke" on the display of a thermal camera. It should be mentioned that only detection of a certain gas can be achieved without the possibility of quantitative analysis. AIRIS module was paired up with LWIR thermal camera (256x256 pixel $\mathrm{HgCdTe}$ array detector) and mounted aboard an UH helicopter to test its capabilities in detecting terrorist arracks involving chemical pollution of urban areas (Fig. 3). 


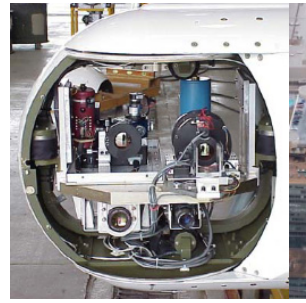

(a)

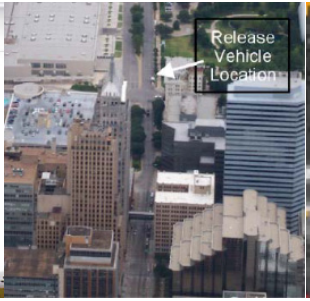

(b)

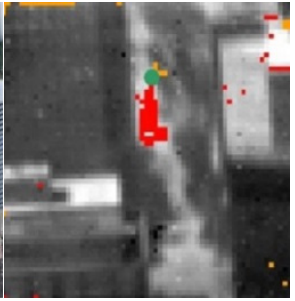

(c)

Figure 3: Application of AIRIS LW and test results: (a) FAT BOY device with AIRIS-LW module aboard an Uh helicopter, (b) truck in the street of Oklahoma City releasing a compound simulating chemical pollution, (c) visualization of the detected SF6 compound [3].

In another solution AIRIS module was used in Wide Area Detector (AIRISWAD). Directional detection capabilities of this system are $360^{\circ}$ in azimuth and $60^{\circ}$ in elevation. High sensitivity of $1 \mu \mathrm{W} /\left(\mathrm{cm}^{2} \mathrm{sr} \mu \mathrm{m}\right)$ and lens design make it possible to detect gas concentrations of $100 \mathrm{ppm}$ from over 1 kilometre distance [3].

Flir Systems offers a range of thermal cameras for gas detection known under GasFindIR brand (Fig. 4(a)). These are devices intended to detect gas leakages in industrial installations (e.g. pipelines in oil refineries). GasFindIR operates using a set of optical filters with spectral characteristics matched to IR absorption bands of selected gases. Currently there are two devices available for the detection of different types of gases - GasFindIR SW (spectral range 3-5 $\mu \mathrm{m}$ ) and GasFindIR LW (spectral range $8-12 \mu \mathrm{m}$ ). Each device can detect about 20 various gases. The gas cloud in the camera image is shown in different color (Fig. 4(b)). These devices, however, cannot identify gas type but they are only able to detect the presence of a pre-selected chemical compound. On the other hand, light weight and simple operation is definitely an asset.

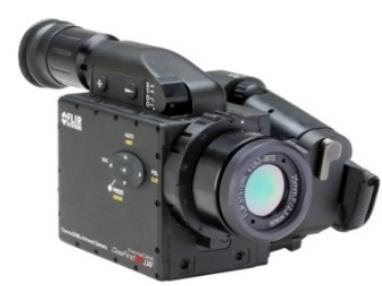

(a)

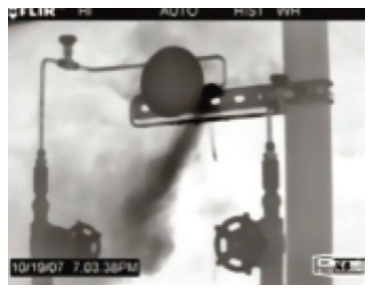

(b)

Figure 4: GasFindIR camera made by Flir Systems, methane leak recorded by GasFindIR camera.

An infrared systems used for stand-off gas detection are also based on Fouriertransform spectrometers. That instruments, particularly well-suited to remote 
detection of chemical compounds, provide excellent estimates of quantitative data. Many authors have presented how the conventional (non-imaging) FTS could be used to perform a quantification of distant gas emissions. Amongst others, the important contributions made by Harig and Matz [4] and Harig et al. [5] should be mentioned. His group performed many measurement campaigns with excellent results, mainly by ensuring proper modelling of the scene and by taking into account the signature of the instrument used. Other groups developed similar approaches, however limiting their study to optically thin plumes [6,7], to dedicated instruments performing optical subtraction [8, 9], or introducing Bayesian algorithms to maximize the use of a priori information [10]. On the other hand, detection and quantification activities with an imaging Fourier-transform spectrometer (IFTS) have been first presented in [11, 12].

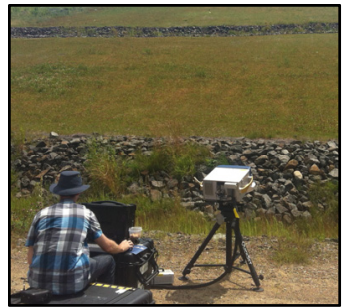

(a)

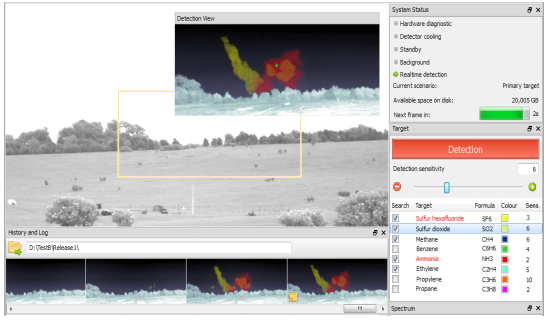

(b)

Figure 5: The infrared imaging Fourier-transform spectrometer (IFTS) HyperCam during the field experiment (a); results of detection and identification Sulfur Dioxide $\mathrm{SO}_{2}$ and Acetone $\mathrm{C}_{3} \mathrm{H}_{6} \mathrm{O}$ (b) $[13,14]$.

Advantages of using the FTIR sensor over a grating based (filter) system include higher resolution for equal cost and the absence of misalignment of different color images due to platform motion. However, FTIR does have the disadvantage of producing a slower frame rate than filter based systems because twice as many points are taken for the same number of spectral points. For an atmospheric tracking of gases however, the HyperCam sensor has a sufficient frame rate $[14,15]$. HyperCam can be used for remote gas detection, identification and quantification from turbulent stack plumes which makes it also suitable to detect terrorist attacks involving the release of hazardous chemical compounds [14-17].

\section{Imaging Fourier Transform Spectroradiometer}

The infrared imaging Fourier-transform spectrometer (IFTS) - Hyper-Cam LWIR - used on experiment was built by Telops Inc. This IFTS use 320x256 pixel Mercury Cadmium Telluride (MCT) focal plane arrays (FPA) with a $6^{\circ} \times 5^{\circ} \mathrm{FOV}$. The FPA has Stirling cooler to provide good noise figures in a field ready package. Spectral information is obtained using a technique called Fourier Transform 
Infrared Radiometry (FTIR). FTIR is a classical interference based technique applied to gas spectroscopy that uses a Michelson interferometer to mix an incoming signal with itself at several different discrete time delays. The resulting time domain waveform, called an interferogram, is related to the power spectrum of the scene through the Fourier transform. An interferogram for each pixel in an image is created by imaging the output of the interferometer onto a focal plane array and collecting data at each discrete time delay. Advantages of using an FTIR sensor over filter or grating based system include higher resolution for equal cost and the absence of misalignment of different color images due to platform motion. However, FTIR does have the disadvantage of producing a slower frame rate than filter based systems because twice as many points are taken for the same number of spectral points. For atmospheric tracking of gases however, the LWIR sensor has sufficient frame rate [16].

Data for this collection can be taken from $0.25 \mathrm{~cm}^{-1}$ to $150 \mathrm{~cm}^{-1}$ spectral resolution between $830 \mathrm{~cm}^{-1}(12 \mu \mathrm{m})$ and $1290 \mathrm{~cm}^{-1}(7.75 \mu \mathrm{m})$ at a frame rate of $0.2 \mathrm{~Hz}$. In addition to the infrared data, visible imagery was taken using a camera that is boresighted to the IR sensor. Fig. 6(a) shows a picture of a Hyper-Cam LWIR. The sensor is controlled by field computer and data is stored on a RAID drive to guarantee integrity of data [17].

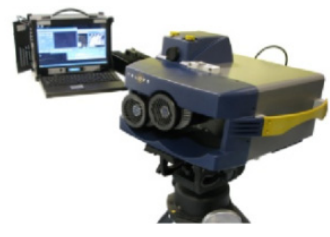

(a)

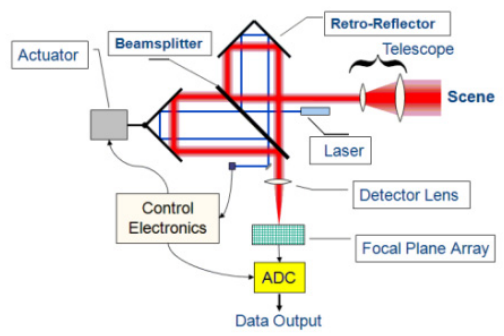

(b)

Figure 6: The infrared imaging Fourier-transform spectrometer (IFTS) HyperCam (a) and block diagram of imaging Fourier-transform spectroradiometer (b).

Imaging Fourier-transform spectroradiometer - HyperCam - uses the layout of a Michelson interferometer. Its schematic diagram, showing the elements responsible for the change of the length of optical path, is shown in Fig. 6(b).

In the presented system the retro-reflectors are used instead of flat mirrors. As a result it is easier to move mirror keeping the reflecting plane in constant position with respect to the optical axis. It should be mentioned here that the interferometer works in the far infrared range, thus the necessary difference in optical paths requires large mirror travel. For such long moves in a classical setup with flat mirrors the skew of mirror plane is difficult to avoid which may result in considerable measurement errors. The solution presented in Fig. 6(b) minimizes such errors. 
The main difference between standard and imaging interferometer is the application of focal plane array (FPA) detector type. The imaging device can be described as classical interferometer multiplied by the number of pixels in the array. As a result the spectral analysis of interferograms at single pixels gives the spectral information of the entire image. The analysis of incident radiation at pixel level is identical for every array element.

The change in amplitude as a result of optical path difference $x$ (this is a double offset retro-reflectors), for $\lambda_{i}$ wavelength. Detector response is proportional to the radiant intensity of incident radiation. The spectral distribution for an entire image is obtained by performing the analysis for every pixel of an FPA array. The results can be treated as 3-dimensional data (two image coordinates and a wavelength datacube).

\section{Method of detection and identification chemical agents}

The target detection and identification algorithm is generally based on three key factors: the composition of the analyzed pixel, the type of model used to estimate the variability of the target and background spaces, and the model used to describe the pure and mixed pixels. The mathematical representation of a mixed pixel depends on whether the background (or target) space is estimated statistically or geometrically. The considered sub-pixel target detection algorithms are of a stochastic nature. When the background is entirely represented by its statistics, the detection problem consists in extracting the targeted spectral signatures from a background noise $\epsilon$.

The clutter matched filter $(C M F)$ is a recognized method and provides a standard by which to compare other detection techniques in the vector-valued observation paradigm. Consider the vector-valued signal model:

$$
r_{t}=z_{t}+e_{t} b
$$

where the pixels in an image are indexed by $\mathrm{t}$ and the background image information is $\mathrm{z}$. The vectors $r_{t}, z_{t}, b$ are $m \times 1$ column vectors. A signal of interest is denoted by b and a signal "strength" is denoted by $e_{t}$. In this situation it may be desired to detect the presence of $b$ among the background information.

The clutter matched filter is an example of an optimal detector for an additive signal on a Gaussian background in vector-valued data. The clutter matched filter is commonly written as

$$
C M F_{t}=\frac{b^{T} K^{-1} r_{t}}{\sqrt{b^{T} K^{-1} b}}
$$

There are several ways to arrive at this expression [19]. One method is to maximize a signal-to-clutter ratio much like the one maximized for Fisher's discriminant. The discriminant function described by Fisher [20] is the oldest classification technique used in this study. The goal of this discriminant is to project multi-class data $\left(x \in X_{i}, i \in\{0,1, \ldots, k\}\right)$ with high dimensionality to a 
low (perhaps one) dimensionality, while maintaining the separation of the classes. This discriminant is developed here in the simplest two-class case, where it is desired to project the data $x$ onto a single dimension (by some $y=w_{T} x$ ) in such a way that maximizes the separation of the two classes. The set of projected data $y$ could then be separated into two groups $Y_{1}$ and $Y_{2}$ that should correspond very closely to the original classes $X_{1}$ and $X_{2}$. Maximizing the separation of these two classes in the lower dimension can be done in a meaningful way by maximizing the difference between class means relative to some description of the variance of the classes.

This method uses to find the maximum of the signal-to-clutter ratio, Lagrange multipliers are used and the minimum of a constrained optimization cost function is found. Another common method is to assume the background data is Gaussian and perform a hypothesis test on the presence of the signal $b$ in the observation. This is the approach that will be taken here.

Let the observations have this probability density function:

$$
f(r)=\frac{1}{(2 \pi)^{m} / \sqrt{|K|}} \exp \left(-\frac{1}{2}(r-\mu)^{T} K^{T}(r-\mu)\right)
$$

where $\mu$ is the mean and $K$ is the covariance. Propose a hypothesis test on the presence of signal in the observation. This really tests for a shift in the mean, as shown in the hypotheses below. Let

$$
\begin{gathered}
H_{0}: \mu=\mu_{z} \\
H_{1}: \mu=\mu_{z}+e b
\end{gathered}
$$

and form the likelihood ratio for this test. This likelihood ratio $(L R)$ can be written as

$$
L R(r)=\frac{\exp \left(-\frac{1}{2}(r-\mu-e b)^{T} K^{-1}(r-\mu-e b)\right)}{\exp \left(-\frac{1}{2}(r-\mu)^{T} K^{-1}(r-\mu)\right)}
$$

It is important to understand that there are many paths that photons can take to arrive at a sensor pointed at the ground. There are photons emitted by materials at their respective temperatures, and transmissions through gasses, as well as absorptions and reflections by opaque objects. Though there are many paths to consider, there are only a few that significantly contribute to the signal measured at the sensor in LWIR measurements. Only those important paths will be considered in this development, which is drawn mainly from the literature focusing on the detection problem, but also from some work done on artificial plume insertion [18, 19, 21-23]. Firstly, a model will be developed for paths that arrive at a sensor which is pointed at the ground, but not viewing any gaseous plumes in the atmosphere. The main contributing paths to the signal in this situation are atmospheric upwelling $\left(L_{u}\right)$, background (ground) radiance $\left(L_{g}\right)$, and 
noise $\left(L_{n}\right)$. Atmospheric upwelling is radiance that comes from the atmosphere's thermal emission at its temperature. The ground radiance is described as a combination of the thermal emission of an object at the ground's temperature scaled by the emissivity of the material emitting $\left(\varepsilon_{g}\right)$, and atmospheric downwelling. The noise term encompasses a few effects including the noise on the focal plane and the thermal emission of the sensor itself onto its focal plane. The combination measured at the sensor can be stated as:

$$
L_{\text {clear }}(\lambda)=L_{u}(\lambda)+L_{g}(\lambda) \tau_{\text {atm }}(\lambda)+L_{n}(\lambda)
$$

which describes the attenuation of the ground radiance by the transmissivity of the atmosphere, $\tau_{\text {atm }}$. This also denotes the wavelength $(\lambda)$ dependence of all these terms. The background radiance must be further broken up into constituent terms:

$$
L_{g}(\lambda)=B\left(\lambda, T_{g}\right) \varepsilon_{g}(\lambda)+L_{d}(\lambda)\left(1-\varepsilon_{g}(\lambda)\right)
$$

The background material is held to be at some temperature $T_{g}$, and it is assumed to be radiating as a perfect blackbody does. The $B\left(\lambda, T_{g}\right)$ function represents the Planck function for radiating blackbodies. This radiation is scaled by the emissivity of the material, $\varepsilon_{g}$. The other significant contribution to the background radiance term is the reflected atmospheric downwelling. The atmosphere, as previously mentioned, radiates upwards towards the sensor, but some of that energy will be radiated towards the ground and consequently reflected back towards the sensor. This radiance will not be perfectly reflected, as some will be absorbed by the material. However, for simplicity's sake, it is assumed that the particles absorbing this downwelled radiance are in local thermodynamic equilibrium [23].

Including the effects of a plume in scene can be done in a few short steps. The paths that govern this model are slightly more complicated than before. The atmospheric upwelling radiance remains a significant contributor to the signal, but the downwelling radiance that is reflected to the sensor now passes through the plume. The plume has a similar effect on the background thermal radiance. The resultant effect on these signals (reflected downwelling and background thermal radiance) is further attenuation based on the transmission of the plume $\left(\tau_{p}\right)$. The radiance of the plume material itself also contributes to the signal model. This radiance $\left(L_{p}\right)$ occurs at the temperature of the plume material, and must pass through (and thus be attenuated by) the intervening atmosphere to reach the sensor. The atmospheric attenuation is assumed applied to the plume at the same strength it is applied to the background thermal radiance path. This is because the plume is assumed to be close to the ground, rather than much closer to the sensor. The result of these effects can be written as

$$
L_{\text {plume }}(\lambda)=L_{u}(\lambda)+L_{g}(\lambda) \tau_{\text {atm }}(\lambda) \tau_{p}(\lambda)+L_{p}(\lambda) \tau_{\text {atm }}(\lambda)+L_{p}(\lambda)
$$

This statement describes many of the important paths and their terms, but what is desired is to express this model with a signal term (and an associated strength) and an additive noise term. The signal term should also be a function of the signature of the chemical being detected, $b(\lambda)$. The linear expression from this 
model that explicitly involves $b(\lambda)$. The transmission of the plume can be expressed as follows:

$$
\tau_{p}(\lambda)=\exp (-c b(\lambda)) \approx 1-c b(\lambda)
$$

where $\mathrm{c}$ is the column density of the gas plume, and the chemical signature of the gas is $b(\lambda)$. This transmissivity is approximately linearized using the assumption that $c b(\lambda)$ is small. The assumption of a small $c b(\lambda)$ must be kept true, otherwise the linear model will be unfaithful to the physical process.

A linear model with an additive noise term can now be realized using the result in (10). By allowing the background, or noise term to be:

$$
n(\lambda)=L_{u}(\lambda)+\left[B\left(\lambda, T_{g}\right) \varepsilon_{g}(\lambda)+L_{d}(\lambda)\left(1-\varepsilon_{g}(\lambda)\right)\right] \tau_{a t m}(\lambda) \tau_{p}(\lambda)+L_{n}(\lambda)
$$

and letting the signal term be $b(\lambda)$ and its associated strength term be

$$
e(\lambda)=c\left[B\left(\lambda, T_{p}\right)-B\left(\lambda, T_{g}\right) \varepsilon_{g}(\lambda)+L_{d}(\lambda)\left(1-\varepsilon_{g}(\lambda)\right)\right] \tau_{a t m}(\lambda) \tau_{p}(\lambda)
$$

the model can expressed linearly as

$$
L_{\text {plume }}(\lambda)=e(\lambda) b(\lambda)+n(\lambda)
$$

These can then be expressed in vector notation by letting each entry indexed by $i$ in the vectors correspond to a $\lambda_{i}$ that is significant because of a sensor being used or some other practical motivation. Then let:

$$
\begin{aligned}
& L_{\text {plume }}=L_{u}+\left[B\left(T_{g}\right) \otimes \varepsilon_{g}+L_{d}\left(1-\varepsilon_{g}\right)\right] \otimes \tau_{a t m}+L_{n}+\ldots \ldots . \\
& \ldots \ldots . . . c b \otimes\left[B\left(T_{p}\right)-\left(B\left(T_{g}\right) \otimes \varepsilon_{g}+L_{d}\left(1-\varepsilon_{g}\right)\right)\right] \otimes \tau_{a t m}
\end{aligned}
$$

and let the linearization assignments be made for these terms in the same way they were made for the wavelength-continuous expressions. These values can now be assigned to the vector-valued variables in (1):

$$
z_{t}=L_{u}+\left[B\left(T_{g}\right) \otimes \varepsilon_{g}+L_{d}\left(1-\varepsilon_{g}\right)\right] \otimes \tau_{a t m}+L_{n}
$$

and in this case, the signal strength term is actually expressed as an element-byelement multiply where $e \otimes b$ results in the signal strength being applied. The value of this strength term is

$$
e=c\left[B\left(T_{p}\right)-\left(B\left(T_{g}\right) \otimes \varepsilon_{g}+L_{d}\left(1-\varepsilon_{g}\right)\right)\right] \otimes \tau_{a t m}
$$

For the purposes of this work, however, the element-by-element scaling is assumed to have been applied to the known spectrum $b$ as a pre-processing step, so only the column density $c$ remains as a signal strength scaling term $e$ (16). The linear model (10) reiterated at the beginning of this section can now be constructed using these assignments. This allows the processing techniques based on these 
linear models to be applied with great success. This development shows that the linear model is a valuable, and effective approximation to the radiometric models. It is also clear that when assumptions about column density being small or the plume being close to the ground are broken, this model may cause algorithm performance to degrade.

The detection and identification of chemical agent is performed through postprocessing of the recorded hyperspectral. Measurement of the background is performed to obtain the covariance matrix of the scene. This covariance is used to calculate the clutter-matched filters for several gas signatures from a library based on PNNL data. On each scene measurement, the background is removed and the calculation of the image of clutter-matched filter scores is performed. To detect and identify a given chemical agent, the score from calculations $C M F$ must exceed a given threshold $[18,23,24]$.

\section{Software for detection and identification of chemical agents}

The measurements on a laboratory test stand were performed in order to determine the minimal gas concentration which can be detected by HyperCam LWIR spectroradiometer using the method detection and identification describe up. The flow from gas cylinder was controlled by a precise gas pressure regulator and large-area blackbody was used to determine the value of thermal contrast between gas cloud and background. The applied blackbody has a radiative area of $11 \times 11$ inch and its temperature difference $\Delta T$ can be set with respect to ambient or temperature value measured by an external sensor. In our case this external sensor was placed at the output of gas nozzle thus the thermal contrast between gas cloud and background (blackbody area) could be precisely set.

The following gases were used during the measurements: methanol, 1-propanol and propane-butane mixture, all of those having absorption lines in the far infrared spectral range. The applied gas pressure regulator provided the controlled gas flow. During the measurement session the flow was set at $5 \mathrm{mg} / \mathrm{s}$ which correspond to gas concentration of $1 \%$ in the measurement zone observed by a HyperCam LWIR. Spectral characteristics of gases were measured for the following values of thermal contrast: $\Delta T=1 \mathrm{~K}, \Delta T=2 \mathrm{~K}, \Delta T=3 \mathrm{~K}$. In order to obtain the best possible accuracy the spectral resolution was set at $0.75 \mathrm{~cm}^{-1}$ whereas the frame rate was $0.3 \mathrm{~Hz}$ and it resulted from the chosen resolution of sub-frame windowing (limited area of an array used during measurements which also reduced the field of view). Each measurement was repeated twice in order to avoid accidental errors. The results obtained from HyperCam LWIR spectroradiometer gave finally the spectral characteristics of selected gases for different measurement conditions. The experimental results showing the measured spectral characteristics of methanol and 1-propanol for different thermal contrast.

Verification of the correct operation of algorithm was conducted by detection and measuring the vapors of selected chemical compounds, such as 1-propanol and methanol. Those substances in liquid state were poured into a flat vessel and a gas cloud was emitted due to evaporation. Thermal contrast was modified by changing the temperature of a blackbody located in the background behind the 
vessel. The concentration results given by tested algorithm were compared with calculated ones derived from analytic description of evaporation process. Results obtained during laboratory tests are presented in Fig. 7. This figure shows the images (calculated from datacube) recorded at wavenumber $\lambda=940 \mathrm{~cm}^{-1}$ outside the methanol absorption band, whereas the image recorded at $\lambda=1033 \mathrm{~cm}^{-1}$ inside methanol absorption band (methanol vapor clearly visible on images extracted from datacube). Spectral characteristics obtained after post-processing analysis of measurement data for a specified measurement point (indicated as a red cross) are presented Fig. 7 below the image of registered scene. The results correspond to the measurements of methanol evaporation depicted on images.
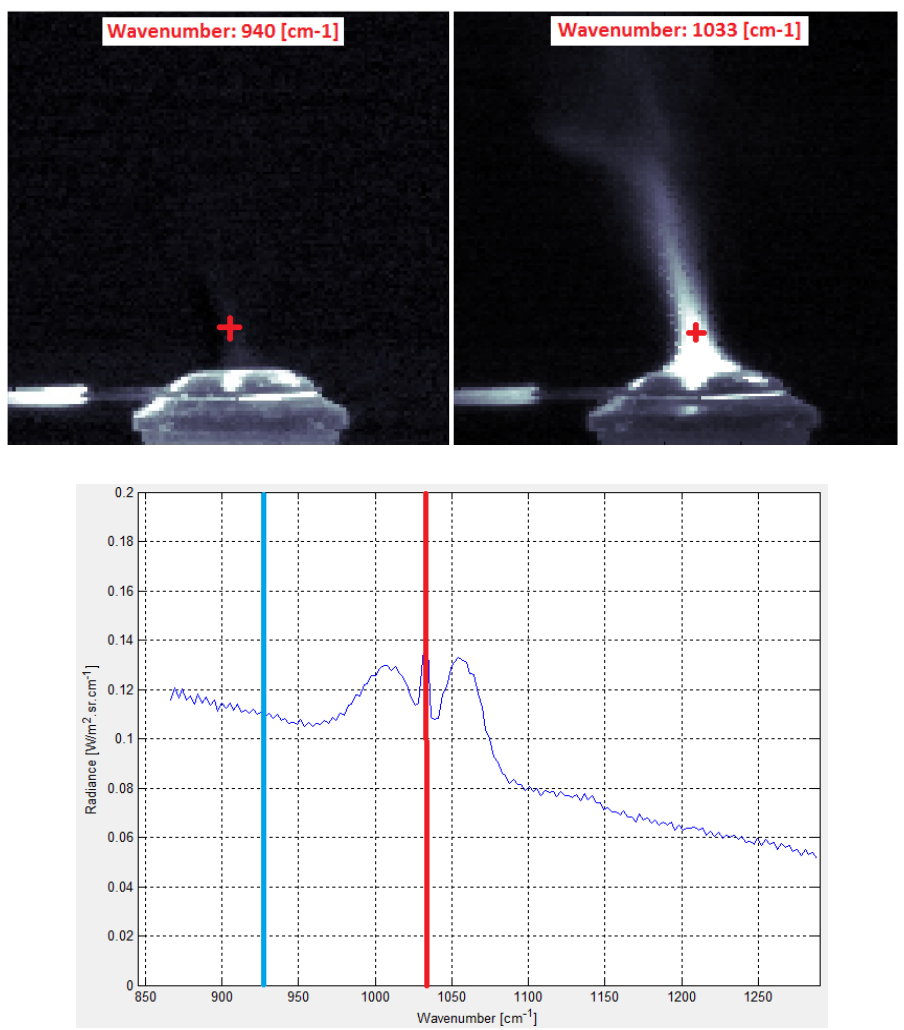

Figure 7: The imaging registered by IFTS HyperCam during laboratory tests.

Method of gas detection and identification (based on CMF and SAM) utilizing hyperspectral data recorded by HyperCam LWIR was implemented in a new software Reveal DRI. This software package is capable of determining experimental conditions, recording of measurement data and automatic gas detection and recognition using internal database of spectral data of chemical compounds. Software takes full, control over spectrometer and its measurement 
settings and is capable of real-time data recording and displaying the image of observed scenery. Processing speed of the software is determined mainly by the spectral resolution of data recorded by spectrometer (the higher is the resolution the longer it takes to process it and visualize results on the screen). Chosen spectral resolution depends also on the spectral properties of the detected compounds narrow absorption bands require higher spectral resolution settings during measurements. Operator can also manually adjusts detection and identification thresholds, which affects the false alarm rate and probability of correct detection.

The new software was tested in laboratory and results are presented on Fig. 8(a).The new software was tested in field conditions in order to verify its correct operation. The following set of chemical compounds was used to test gas detection capability: vapors of 1-propanol and methanol. The results of detection and identification of methanol vapors prove that the gas detection method implemented in Reveal DRI is valid and the software operates correctly (Fig. 8(b)).

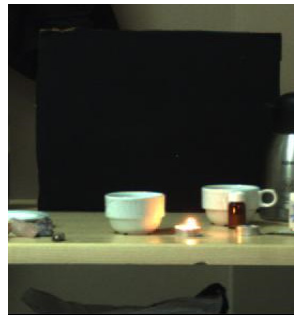

(a)

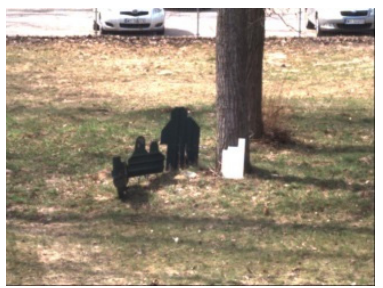

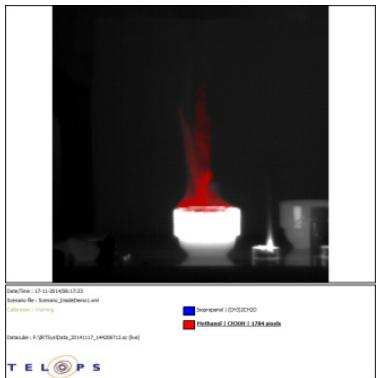

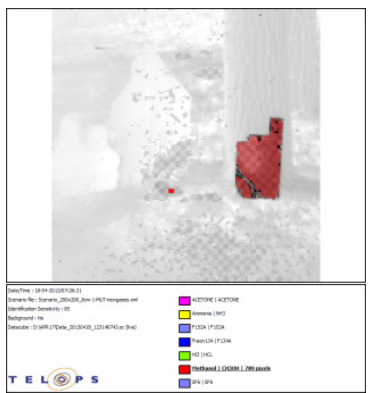

(b)

Figure 8: The image registered by IFTS HyperCam during laboratory tests (a) and field tests (b).

At a distance of $30 \mathrm{~m}$ from IFTS HyperCam has two vessels with methanol and 1-propanol, which as a result of vaporization couples both compounds. During the experiment in the software was selected seven different compounds, which are irrelevant as possible to detect using algorithms implemented in reveal DRI. The results are shown in Fig. 8 (on right images we can find detected methanol which clouds is colored on red color). 


\section{Conclusion}

IFTS is a very useful method for gas detection in the open space. The possibility to present the results in a form of an image is a clear advantage, because the gas can be not only detected, but also located in space. On the basis of conducted experiments it can be stated that the gas concentrations exceeding $1 \%$ can be detected in the open space using the HyperCam spectrometer.

The measurements previously conducted by means of IFTS spectrometers had lower accuracy due to lower sensitivity, the influence of weather conditions (mainly wind speed) and unknown thickness of a visualized gas cloud. This new method combining mathematical analysis, data from reference signature database (PNNL database), CMF (Clutter Matched Filter) module should improve the effectiveness of gas detection by IFTS-based solutions. The new algorithm implemented into new software gives the possibility of detection and identification of very small concentration of gaseous compounds.

\section{References}

[1] Sandsten J., Weibring P., Edner H. and Svanberg S.: Real-time gascorrelation imaging employing thermal background radiation, OPTICS EXPRESS 92, vol. 6, No. 4, 14 February 2000.

[2] Gittins C. M., Marinelli W. J. and Jensen J. O.: Remote Sensing and Selective Detection of Chemical Vapor Plumes by LWIR Imaging FabryPerot Spectrometry, Proceedings of SPIE vol. 4574, pp. 63-71, (2001).

[3] Marinelli W. J., Gittins C. M., Cosofret B. C., Ustun T. E., Jensen J. O.: Development of the AIRIS-WAD Multispectral Sensor for Airborne Standoff Chemical Agent and Toxic Industrial Chemical Detection, presented at 2005 Parallel Meetings of the MSS Specialty Groups on Passive Sensors; Camouflage, Concealment, and Deception; Detectors; and Materials (Charleston, SC), 14-18 February 2005.

[4] Harig, R., Matz, G. (2001). Toxic Cloud Imaging by Infrared Spectrometry: A Scanning FTIR System for Identification and Visualization. Field Analytical Chemistry and Technology, 5(1-2), pp. 75-90.

[5] Harig, R., Matz, G., Rusch, P. (2002). Scanning Infrared Remote Sensing System for Identification, Visualization, and Quantification of Airborne Pollutants. Proc. of SPIE, 4574, pp. 83-94.

[6] Griffin, M.K., Kerekes, J.P., Farrar, K.E., Burke, H.-H.K. Characterization of Gaseous Effluents from Modeling of LWIR Hyperspectral Measurements. Proc. of SPIE, 4381, pp. 360-369 (2001).

[7] Burr, T., Hengartner, N. Overview of Physical Models and Statistical Approaches for Weak Gaseous Plume Detection using Passive Infrared Hyperspectral Imagery. Sensors, 6(12), pp. 1721-1750 (2006).

[8] Lachance, R.L., Thériault, J.-M., Lafond, C., Villemaire, A.J. Gaseous emanation detection algorithm using a Fourier transform interferometer operating in differential mode. Proc. of SPIE, 3383, 124 (1998). 
[9] Thériault, J.-M. Passive standoff detection of chemical vapors by differential FTIR radiometry, Technical Report Defence Research Establishment Valcartier (DREV) TR-2000-156 (2001).

[10] Heasler, P., Posse, C., Hylden, J., Anderson, K. Nonlinear Bayesian Algorithms for Gas Plume Detection and Estimation from Hyper-spectral Thermal Image Data. Sensors, 7, 905-920 (2007).

[11] Spisz, T.S., Murphy, P.K., Carter, C.C., Carr, A.K., Vallières, A., Chamberland, M. (2007). Field test results of standoff chemical detection using the FIRST. Proc. of SPIE, 6554, 655408.

[12] Chamberland, M., Belzile, C., Farley, V., Legault, J.-F., Schwantes, K. Advancements in field-portable imaging radiometric spectrometer technology for chemical detection. Proc. of SPIE, 5416, 63-72 (2004).

[13] Farley V., Chamberland M., Lagueux P., Vallières A., Villemaire A., Giroux J.: Chemical agent detection and identification with a hyperspectral imaging infrared sensor, Proc. of SPIE 6661, 66610L (2007).

[14] Vallières A., Villemaire A., Chamberland M., Belhumeur L., Farley V., Giroux J., Legault J.-F.: Algorithms for chemical detection, identification and quantification for thermal hyperspectral imagers, Proc. of SPIE 5995, 59950G (2005).

[15] Chamberland M., Belzile C., Farley V., Legault J.-F., Schwantes K.: Advancements in field-portable imaging radiometric spectrometer technology for chemical detection, Proc. of SPIE 5416, pp. 63-72 (2004).

[16] M. Kastek, T. Piątkowski, P. Trzaskawka, Infrared imaging Fourier transform spectrometer as the stand-off gas detection systems, Metrology and Measurement Systems, Vol. XVIII, No. 4, pp. 607-620 (2011).

[17] Kastek M., Piątkowski T., Polakowski H.; Infrared imaging Fouriertransform spectrometer used for standoff gas detection, 19th International Conference on Modelling, Monitoring and Management of Air Pollution, WITPress, Southampton, Boston, UK, pp. 161-172-237 (2011).

[18] M. Kastek, T. Piatkowski, M. Zyczkowski, M. Chamberland, P. Lagueux, V. Farley, Hyperspectral Imaging Infrared Sensor Used for Enviromental Monitoring, Acta Physica Polonica A, vol. 124 (3), pp. $463-467$ (2013).

[19] Theiler, J., Foy, B. R., \& Frasner, A. M., Beyond the adaptive matched filter: nonlinear detectors for weak signals in high-dimensional clutter. Proc. SPIE, vol. 6565, no. 3 (2007).

[20] Fisher, R., The use of multiple measurements in taxonomic problems. Annals of Eugenics, vol. 7, pp. 179-188 (1936).

[21] Czerwinski, R. N., Upham, C. A., E. C. Wack, E. C., Burke, K., \& Griffin, M. K. A procedure for embedding effluent plumes into LWIR imagery. Proc. SPIE, vol. 5806, no. 1, pp. 78-87 (2005).

[22] O’Donnell, E. M., Messinger, D. W., Salvaggio, C., \& Schott, J. Identification and detection of gaseous effluents from hyperspectral imagery using invariant algorithms. Proc. SPIE, vol. 5425, pp. 573-582 (2004). 
[23] Kuo, S. D., Schott, J. R., \& Chang, C. Y. Synthetic image generation of chemical plumes for hyperspectral applications. Optical Engineering vol. 39, no. 4, pp. 1047-1056 (2000).

[24] Kastek, M., Piątkowski, T., Dulski, R., Chamberland, M., Lagueux, P., Farley, V., Method of gas detection applied to infrared hyperspectral sensor, Phot. Lett. of Poland, 4 (4), 146 (2012). 